\title{
Electrophysiological and oviposition responses of Asian corn borer, Ostrinia furnacalis (Lepidoptera: Crambidae), to compounds rinsed from the surfaces of sugarcane and maize leaves
}

\author{
Xing-Chuan JIANG*, Wen-Xia DONG, Bin ChEN, Chun XiAO, Fu-Rong GUI, Nai-Sheng YAN, Lei QIAN \\ and ZHENG-Yue LI** \\ College of Plant Protection, Yunnan Agricultural University, Agro-Biodiversity and Pest Management of Education Ministry \\ of China, Yunnan Key Laboratory Agro-Biodiversity and Conservation, Kunming 650201, China; \\ e-mails: lizhengyue@ynau.edu.cn; jxc678@sina.cn; dongwenxia@163.com; chbins@163.com; ynaisheng@163.com
}

Key words. Lepidoptera, Crambidae, Ostrinia furnacalis, GC-EAD, oviposition, sugarcane, maize, leaf surface

\begin{abstract}
The Asian corn borer, Ostrinia furnacalis, is one of the most important pests of maize (Zea mays) in China. However, the mechanism by which intercropping sugarcane (Saccharum officinarum) and maize controls this pest via compounds rinsed from leaf surfaces is still unclear. Electrophysiological and oviposition responses of the Asian corn borer to compounds rinsed from leaf surfaces of sugarcane and maize were studied. Coupled gas chromatographic-electroantennographic detection (GC-EAD) analyses revealed that O. furnacalis responds to 21 compounds, which are present in both the compounds rinsed from leaf surfaces of sugarcane and maize. The effects of these compounds in concentrations of $5 \times 10^{-3} \mathrm{~g} / \mathrm{mL}$ or $5 \times 10^{-4} \mathrm{~g} / \mathrm{mL}$ on female oviposition behaviour were tested. They were attracted to oviposit on substrates coated with most of the test compounds, except nonanal. It is suggested that the compounds from the leaf surfaces of sugarcane induced $O$. furnacalis females to oviposit because most of them are similar to maize's leaf surface compounds, which results in a reduction in the number of egg masses laid on maize leaves.
\end{abstract}

\section{INTRODUCTION}

The Asian corn borer, Ostrinia furnacalis Guenée (Lepidoptera: Crambidae), is one of the most serious insect pests of maize (Zea mays L.) in China (He et al., 2006; Guo \& $\mathrm{Li}, 2009)$. It is distributed from China to Australia and occurs in the Solomon Islands (Nafus \& Schreiner, 1991). In northern parts of its range, it generally produces one (or, in some cases, multiple) generation per year, but in the tropics (or in the laboratory), generations are continuous and overlapping (Nafus \& Schreiner, 1991). O. furnacalis neonates tend to feed on the tassels of maize, then burrow into the stalk and damage the whole plant and may cause a 20\% to $80 \%$ reduction in yield each year in some areas (Zhou et al., 1995). This generalist herbivore also attacks other economic crops, such as bell pepper (Capsicum annuum L.), ginger (Zingiber officinale Roscoe), sorghum [Sorghum bicolor (L.) Moench] and cotton (Gossypium hirsutum L.) (Nafus \& Schreiner, 1991). Insecticides are still the main way of controlling $O$. furnacalis in most maize growing areas, though it is difficult to control since its larvae tunnel into the plant (Nafus \& Schreiner, 1991). Accepting the need to reduce pesticide residues an economic and ecological way needs to be developed to control this pest (Guo \& Li, 2009).

Based on farming experience intercropping is an effective method of controlling diseases and insect pests and can also boost yields and reduce the incidence of disease (Risch et al., 1983; Zhu et al., 2000; Li et al., 2009). In- tercropping maize with various other crops also results in increases in yield (Li et al., 2009), nitrogen utilization (Eaglesham et al., 1981; Jellum \& Kuo, 1996) and increases in the quantity and quality of forage (Javanmard et al., 2009). Maize has been successfully used in biocontrol and for increasing yield in intercropping systems. Economic crops and grass are intercropped with maize as trap crop (Shelton $\&$ Badenes-Perez, 2006) to reduce the numbers of pests on maize. In New Zealand mustard is a good trap crop intercropped with maize for managing the green vegetable bug (Nezara viridula) (Rea et al., 2002). Maize is successfully intercropped with tomato, resulting in higher net income due to high yields of tomato and a stable yield of maize. Calumpang \& Navasero (2013) report the results of a study on the oviposition behaviour of $O$. furnacalis and the Earwig (Euborelia annulipes) in an intercropping system consisting of selected crops and weeds with sweet maize. Their results indicate that eggplant (Solanum melongena) is a good companion crop for sweet maize because it deters oviposition, which results in fewer egg masses being laid on maize. Bao \& Lin (2011) report that intercropping sugarcane (Saccharum officinarum) and maize results in an increase in the yield of corn and no reduction in the yield of sugarcane. This successful intercropping system is now widely used in both Guangxi and Yunnan Provinces in China.

However, to the best of our knowledge, there is little research on the ecological function of plant volatiles in inter-

\footnotetext{
* Present address: College of Plant Protection, Anhui Agricultural University, Hefei 230036, China.

** Corresponding author.
} 
cropping systems with maize. Plant volatiles have crucial roles in mediating insect behaviour such as host plant location and acceptance by phytophagous insects, attracting parastoids or predators of insect pests (Khan et al., 1997), inducing insects to oviposit and influencing plant-plant interactions (Huang et al., 2009; Konstantopoulou et al., 2004; Steinbauer et al., 2004; Ruther \& Kleier, 2005). It is proposed that intercrops fall in two functional groups, trap plants and repellent plants, which have chemical characteristics that make them attractive or repellent to certain insect pests, thereby reducing pest pressure on the main crop (Khan et al., 2000; Poveda \& Kessler, 2012). Ethological control by applying infochemicals to disrupt feeding, mating and oviposition behaviour may have an important role in such a system (Shelton \& Badenes-Perez, 2006; Cook et al., 2007; Zehnder et al., 2007; Guo \& Li, 2009). Pilot experiments on the physiology of $O$. furnacalis indicate it can detect oviposition-deterring fatty acids and methyl esters (Guo \& Li, 2009). The chemicals in the epicuticular wax of maize may affect the oviposition behavior of the European corn boer (Ostrinia nubilalis) (Udayagiri \& Mason, 1997).

Resource concentration hypothesis suggests that intercropping may distract $O$. furnacalis by interfering with the chemical or visual cues emanating from maize and the intercrop plants (Litsinger et al., 1991). A crude extract of the compounds in the surfaces of leaves of maize is attractive to $O$. furnacalis moths, but that of sugarcane (S. officinarum) leaves repels their first instar larvae and reduces their survival in sugarcane-maize intercropping systems (Jiang et al., 2014) . However, the real reason why sugarcane acts as a trap crop is unclear, but it does reduce the numbers of O. furnacalis ovipositing on maize leaves. Does sugarcane have leaf surface compounds that are chemically similar to maize and do they affect the oviposition behaviour of O. furnacalis? Or, are there special chemicals in sugarcane leaf surface compounds that deter $O$. furnacalis females from ovipositing? In the present study, compounds rinsed from leaf surfaces of sugarcane and maize were compared and the electrophysiological and oviposition behavioural responses of $O$. furnacalis to the leaf surface compounds of sugarcane and maize were studied in order to determine whether its oviposition behaviour is likely to be affected by sugarcane in intercropping systems.

\section{MATERIAL AND METHODS}

\section{Plants}

Sugarcane (variety Xintaitang-22) and maize (variety $\mathrm{Hu}-$ idan-4) varieties used in this study are both commonly planted varieties in Yunnan Province, China. The method of cultivating these plants was a modified version of that used by Bao \& Lin, 2011, as detailed below. Sugarcane seeds were germinated in incubators (LTC-450, SANTN, China, at $26.0^{\circ} \mathrm{C} \pm 1.0$, under a $14 \mathrm{~L}: 10 \mathrm{D}$ photoperiod, $70.0 \% \pm 10.0$ relative humidity and 25,000 LX illumination intensity). Ten days later, seedlings with similar growth rates were transplanted into a greenhouse $(8.0 \times 12.0 \mathrm{~m})$. The interval between rows was $1 \mathrm{~m}$ and interval between individual plants in a row was $25 \mathrm{~cm}$. Then, the seedlings were covered with a white, thin plastic membrane. 30 days later, maize seeds were seedling in the incubators, and maize shoots with a similar growth rate were transplanted into a greenhouse five days after emergence. Maize seedlings were intercropped with sugarcane in the same pattern. During the experiment no chemicals were applied to the plants.

\section{Insects}

Adults of $O$. furnacalis were collected from a maize field of the Yunnan Agricultural University, Kunming. The larvae were fed on a semi-artificial diet (Zhou et al., 1980) in incubators (at $26.0^{\circ} \mathrm{C} \pm 1.0$, under a $14 \mathrm{~L}: 10 \mathrm{D}$ photoperiod, $70.0 \% \pm 10.0 \%$ relative humidity and 25,000 LX illumination intensity) for several generations. Adults were fed a $10.0 \%(\mathrm{v} / \mathrm{v})$ honey solution (honey mixed with distilled water). The pupae were individually placed in finger tubes $(3.0 \mathrm{~cm}$ in diameter and $10.0 \mathrm{~cm}$ in height) and later, the emerged healthy adults were used in the experiments.

\section{Collection of leaf surface compounds}

Leaf surface compounds were collected using a modified version of Udayagiri \& Mason's (1995) method. One hundred grams of the $2^{\text {nd }}$ and $4^{\text {th }}$ leaves from the tops of sugarcane and maize plants were cut off and separately washed in dichloromethane (99.99\%, Tjshield, China, http://www.tjshield.com). The extracts were filtered twice through filter paper. For quantitative analyses, $0.5 \mu \mathrm{g}$ Nonanoic acid ethyl ester (99\%, TCI, Japan) was added as an internal standard to each sample. This compound was not detectable in the extracts of sugarcane and maize leaves in the preliminary analyses. Each sample was reduced to $100 \mu \mathrm{L}$ under a gentle stream of $\mathrm{N}_{2}$, and then stored at $-4^{\circ} \mathrm{C}$ until required for the physiological and behavioural tests. The chemicals in the sugarcane and maize leaf extracts were analyzed using GC-MS and GC-EAD.

\section{Electrophysiological experiments}

Compounds rinsed from the surfaces of leaves were analyzed using an Agilent Technologies HP 7890A gas chromatograph, equipped with a HP-5MS column $(30 \mathrm{~m} \times 0.25 \mathrm{~mm} \times 0.25 \mu \mathrm{m})$, coupled to an electroantennographic detector (EAD, Syntech, Germany). The initial oven temperature was $40^{\circ} \mathrm{C}$, programmed to increase at $3^{\circ} \mathrm{C} / \mathrm{min}$ to $80^{\circ} \mathrm{C}$, then $5^{\circ} \mathrm{C} / \mathrm{min}$ to $260^{\circ} \mathrm{C}$ and then held at this temperature for $15 \mathrm{~min}$. Nitrogen was used as carrier gas $(1.0 \mathrm{~mL} / \mathrm{min})$. All signal sources were connected to a stimulus controller (CS-55, Germany) and then connected to a serial data acquisition interface (IDAC-2, Germany). Antennae were excised from the heads of $O$. furnacalis adults using sharp scissors. A few segments from the tips of antennae were cut off and mounted on the antennae holder with two metal electrodes using conductive gel (Spectra 360, America), and then the electrode holder was inserted into the EAD probe. Testing began after a relatively-stable baseline was achieved. The outlet of the GC column was split in a $1: 1$ ratio between the flame ionization detector (FID) and the electroantennographic detector, a piece of antennae of $O$. furnacalis. The mounted antennae were positioned in the charcoalfiltered and humidified air steam that carried the VOCs eluted from the GC column. The antennal and FID signals were amplified and recorded simultaneously using Syntech software (GCEAD, version 4.6, Syntech, Germany). This method is a modified version of that used by Lu et al. (2012). Each sample was tested 10 times. Identities of EAD-active compounds were verified by comparison of mass spectra and retention times with those of synthetic standards. These compounds were nonanal (95\%, TCI), decanal $(95.0 \%, \mathrm{TCI})$, pentacosane $(97.0 \%, \mathrm{TCI})$, hexacosane $(95.0 \%, \mathrm{TCI})$, heptacosane $(95.0 \%, \mathrm{TCI})$, octacosane $(98.0 \%$, TCI), nonacosane $(98.0 \%$, TCI), tetradecanoic acid $(99.5 \%$, Dr. Ethrenstorfer $\mathrm{GmbH})$, hexadecanoic acid $(97.5 \%$, J\&K), (Z)9-octadecenamide $(98.5 \%$, J\&K), (Z)-13-docosenamide (98.0\%, $\mathrm{J} \& \mathrm{~K})$, hexadecane $(98.5 \%$, J\&K), heptadecane $(98.5 \%$, J\&K), 

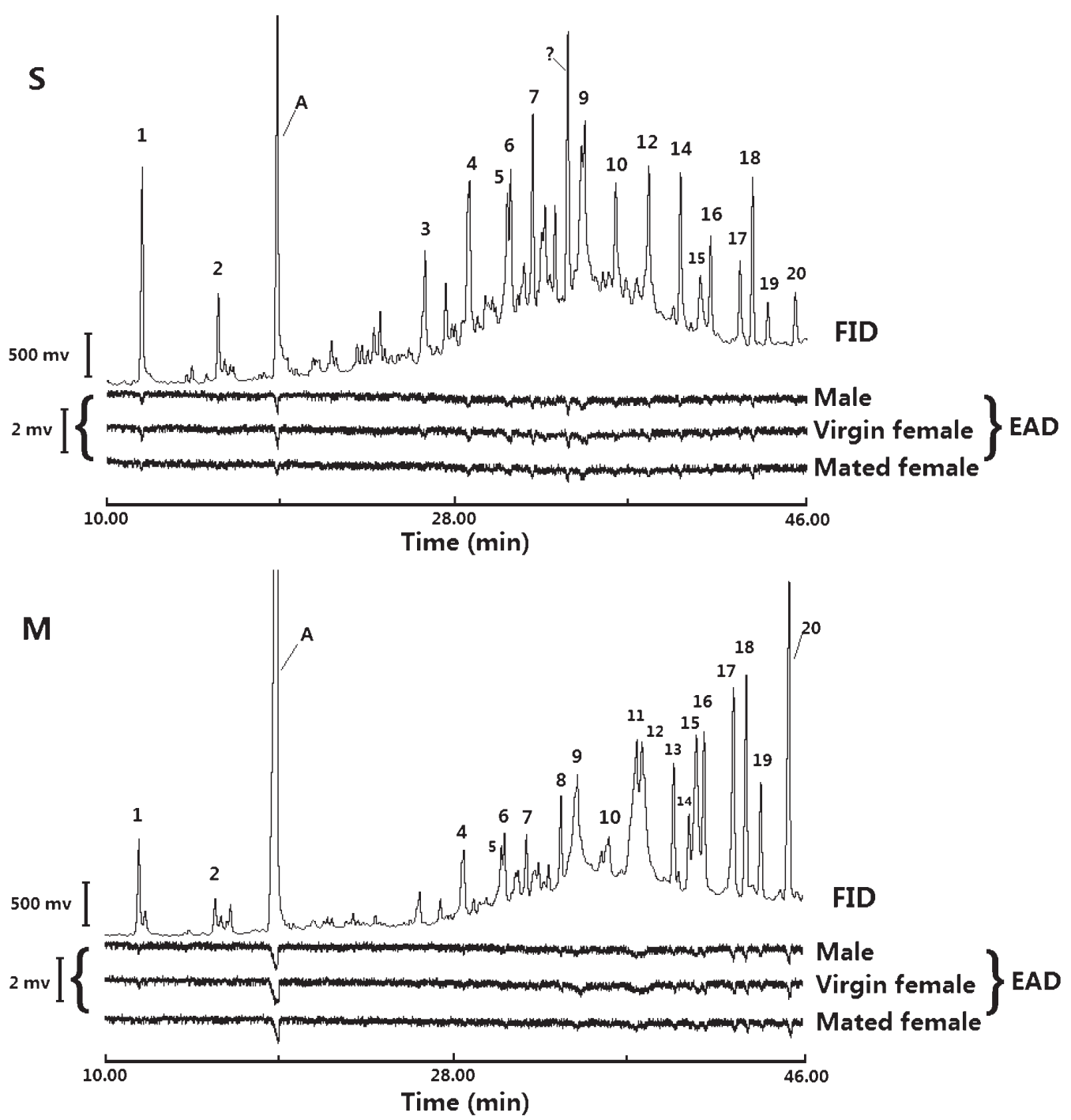

Fig. 1. The GC-EAD responses of Ostrinia furnacalis moths to leaf extracts of sugarcane (S) and maize (M), respectively. The upper trace is the flame ionization detector response (FID) and the lower trace the antennal response of males, virgin females and mated females (EAD). Consistently EAD-active compounds were: A - Internal standard Nonanoic acid, ethyl ester; ? - Unknown; 1 - Nonanal; 2 - Decanal; 3 - Hexadecane; 4 - Heptadecane; 5 - Tetradecanoic acid; 6 - Octadecane; 7 - Hexadecanoic acid methyl ester; 8 - (Z)7-Hexadecenoic acid; 9 - Hexadecanoic acid; 10 - Octadecanoic acid; 11 - Docosane; 12 - Tricosane; 13 - (Z)-9-Octadecenamide; 14 - Tetracosane; 15 - Pentacosane; 16 - Hexacosane; 17 - Heptacosane; 18 - (Z)-13-Docosenamide; 19 - Octacosane; 20 - Nonacosane.

octadecane $(98.0 \%, \mathrm{~J} \& \mathrm{~K})$, docosane $(99.0 \%, \mathrm{~J} \& \mathrm{~K})$, tricosane (98.0\%, J\&K), octadecanoic acid (98.0\%, ULTRA Scientific), hexadecanoic acid methyl ester (99.90\%, ULTRA Scientific), and tetracosane $(99.0 \%$, Acros Organics). Each antenna tested was obtained from a different adult. The compounds rinsed from the surfaces of the leaves of both sugarcane and maize were tested on male moths, virgin females and mated female moths.

\section{Coupled gas chromatography-mass spectrometry analysis}

Samples $(2 \mu \mathrm{L})$ were analyzed on a capillary GC column (HP$5,30 \mathrm{~m} \times 0.25 \mathrm{~mm} \times 0.25 \mu \mathrm{m})$ directly coupled to a mass spectrometer (Agilent 5973). Ionization was achieved by electron impact at $70 \mathrm{eV}, 250^{\circ} \mathrm{C}$. The temperature program was as described above. GC-EAD-active compounds were identified by comparing their mass spectra with NIST library spectra (Agilent Technologies, USA) and confirmed using authentic reference compounds, except for (Z)-7-hexadecenoic acid, which was confirmed using Kovats indics (KI)

\section{Oviposition bioassays}

Oviposition bioassays were based on those used in previous studies (Udayagiri \& Mason, 1995; Jiang et al., 2014). Experiments were done using two day-old and mated females, at 9:00 pm each day. Five females were kept in a cylinder-shaped oviposition cage $(10 \mathrm{~cm}$ diameter and $20 \mathrm{~cm}$ in height $)$ with a ball of absorbent cotton wool soaked in honey water to provide a source of food for $O$. furnacalis. The oviposition cage consisted of two pierced circular plastic plates $(10 \mathrm{~cm}$ in diameter) and four straight iron wires ( $1 \mathrm{~mm}$ diameter and $20 \mathrm{~cm}$ in length). When the experiment started, the two circular plastic plates were covered with plastic film (Miaojie, China). These four steels would be closed with a piece of gauze. The cages were placed in a dark chamber (temperature $26.0^{\circ} \mathrm{C} \pm 1.0, \mathrm{RH} 70.0 \pm 10.0 \%$ ). Eggs laid on the plastic films were counted the next day at 7:00 am.

EAD-active chemicals were purchased (see above) and tested individually in dual-choice assays, as above, except no standard was available. The synthetic standards were diluted with hexane to concentrations of $5 \times 10^{-3} \mathrm{~g} / \mathrm{mL}$ and $5 \times 10^{-4} \mathrm{~g} / \mathrm{mL}$, respective- 
TABLE 1. Compounds in leaf extracts of sugarcane and maize. Italicized compounds were chosen for behavioural tests because they were detected by the antennae of $O$. furnacalis using GC-EAD.

\begin{tabular}{|c|c|c|c|c|}
\hline \multirow{2}{*}{ Compounds } & \multicolumn{2}{|c|}{ GC-MS } & \multicolumn{2}{|c|}{ GC-EAD active } \\
\hline & Sugarcane & Maize & Sugarcane & Maize \\
\hline Acetic acid butyl ester & + & + & & \\
\hline 3-Penten-2-ol & + & + & & \\
\hline (E)-2-Hexenal & & + & & \\
\hline (Z)-3-Hexene-1-ol & + & + & & \\
\hline Nonane & + & + & & \\
\hline n-Heptanal & + & + & & \\
\hline Hexanoic acid & & + & & \\
\hline Octanal & + & + & & \\
\hline (Z)-3-Hexenyl acetate & + & + & & \\
\hline (Z)-Ocimene & & + & & \\
\hline Nonanal & + & + & + & + \\
\hline Octanoic acid & & + & & \\
\hline$\alpha$-Terpineol & + & + & & \\
\hline Decanal & + & + & + & + \\
\hline 2-Methyl octanoic acid ethylester & + & & & \\
\hline Nonanoic acid & + & + & & \\
\hline Decanoic acid & & + & & \\
\hline Tetradecane & + & + & & \\
\hline Isolongifolene & + & + & & \\
\hline (E)-6,10,Dimethyl-5,9-undecadien-2-one & + & + & & \\
\hline Pentadecane & + & + & & \\
\hline Dodecanoic acid & + & + & & \\
\hline Hexadecane & + & + & + & \\
\hline 2,6,10-Trimethyl-pentadecane & + & + & & \\
\hline Heptadecane & + & + & + & + \\
\hline $2,6,10,14$-Tetramethyl pentadecane & + & + & & \\
\hline Tetradecanoic acid & + & + & + & + \\
\hline Octadecane & + & + & + & + \\
\hline 2,6,10,14-Tetramethyl hexadecane & + & + & & \\
\hline Isopropyl myristate & + & & & \\
\hline Neophytadiene & + & + & & \\
\hline 6,10,14-Trimethyl-2-pentadecanone & & + & & \\
\hline Pentadecanoic acid & + & + & & \\
\hline Cyclotetradecane & + & & & \\
\hline Nonadecane & + & + & & \\
\hline Hexadecanoic acid methyl ester & + & + & + & + \\
\hline (Z)-7-Hexadecenoic acid & + & + & & + \\
\hline Unknown & + & & + & \\
\hline Hexadecanoic acid & + & + & + & + \\
\hline Eicosane & + & + & & \\
\hline Heneicosane & + & + & & \\
\hline 9-Octadecenoic acid & + & & & \\
\hline Octadecanoic acid & + & + & + & + \\
\hline 2-Propenoic acid, 3-(4-methoxyphenyl)-, 2-ethylhexyl ester & + & & & \\
\hline Docosane & + & + & & + \\
\hline Tricosane & + & + & + & + \\
\hline 4,8,12,16-Tetramethylheptadecan-4-olide & + & & & \\
\hline (Z)-9-Octadecenamide & + & + & & + \\
\hline Tetracosane & + & + & + & + \\
\hline Pentacosane & + & + & + & + \\
\hline 2-Pentacosanone & + & & & \\
\hline Hexacosane & + & + & + & + \\
\hline Heptacosane & + & + & + & + \\
\hline (Z)-13-Docosenamide & + & + & + & + \\
\hline Octacosane & + & + & + & + \\
\hline Nonacosane & + & + & + & + \\
\hline 2-Heptacosanone & & + & & \\
\hline Triacontane & + & + & & \\
\hline
\end{tabular}


ly. Before the experiment, $200 \mu \mathrm{L}$ of synthetic standard solution (each standard was diluted in hexane) was placed on a plastic film and $200 \mu \mathrm{L}$ hexane (98\%, Merck, German) as a control on the other plastic film in a cage, and each $200 \mu \mathrm{L}$ of standard solution contained either $1,000 \mu \mathrm{g}$ or $100 \mu \mathrm{g}$ as used in subsequent behavioural tests, modified after Udayagiri \& Mason (1997), and each synthetic standard paired with a control and repeated 10 times. Oviposition stimulation index (OSI) was calculated using the following formula to determine whether the chemicals tested repelled or attracted females to oviposit:

$O S I=\frac{T-C}{T+C} \times 100$

In this equation, $\mathrm{T}$ is the number of eggs on the treated film and $\mathrm{C}$ the number on the control film. The OSI ranged from -100 to 100 , and when OSI $=0$, it means that the chemical tested was equal to the control; when OSI $<0$, it indicates that the chemical tested was a repellent. Finally, when OSI $>0$, the chemical tested was attractive to the females.

\section{Data analysis}

To equalize the numbers of ovipositions, the results of ten trials of each of the chemicals were transformed into a percentage. The mean percentage that oviposited on the chemically treated and control sides, respectively, were determined. Chi-square tests were used to determine if mated females of $O$. furnacalis were attracted or repelled by the compounds rinsed from the surfaces of the leaves of sugarcane and maize. And the OSIs were used to draw the figures.

\section{RESULTS}

\section{Antennal responses (GC-EAD)}

Fifty eight compounds washed from leaf surfaces were detected using a coupled GC-electrophysiology system, of which 43 compounds (74.14\%) were present in water used to wash both sugarcane and maize leaves, respectively. A total of 21 compounds were detected by antennae of $O$. furnacalis, 18 compounds from sugarcane and 20 from maize. Twenty compounds were chemically characterized, hexadecane in sugarcane extract, $(Z)$-7-hexadecenoic acid and docosane in the maize extract; whereas hexadecane was not detected in the maize extract, and $(Z)$-7-hexadecenoic acid and docosane in the sugarcane extract. These compounds belong to different chemical classes: aldehydes (nonanal and decanal), acids [tetradecanoic acid, (Z)-7-hexadecenoic acid, hexadecanoic acid and octadecanoic acid], esters (hexadecanoic acid methyl ester), amides [(Z)-9-octadecenamide and (Z)-13-docosenamide] and hydrocarbons (from C16 to C18, and C22 to C29) (Fig. 1 and Table 1).

\section{Oviposition bioassays}

The baseline of hexane compared to hexane and a blank control in the oviposition cages was tested, which indicated that $O$. furnacalis showed no bias when ovipositing on solvent control film $(48.50 \pm 6.57 \%)$ and blank control film $\left(47.22 \pm 7.62 \%, \chi^{2}=0.366, P=0.723\right)$.

Most of the compounds rinsed from leaf surfaces of sugarcane and maize were attractive to $O$. furnacalis, whereas tetradecanoic acid (100 $\mu \mathrm{g}: \chi^{2}=14.572, P=0.000 ; 1,000$ $\left.\mu \mathrm{g}: \chi^{2}=15.040, P=0.000\right)$, hexadecanoic acid $(100 \mu \mathrm{g}$ : $\left.\chi^{2}=9.105, P=0.003 ; 1,000 \mu \mathrm{g}: \chi^{2}=4.230, P=0.04\right)$, octadecanoic acid (100 $\mu \mathrm{g}: \chi^{2}=4.849, P=0.028 ; 1,000$
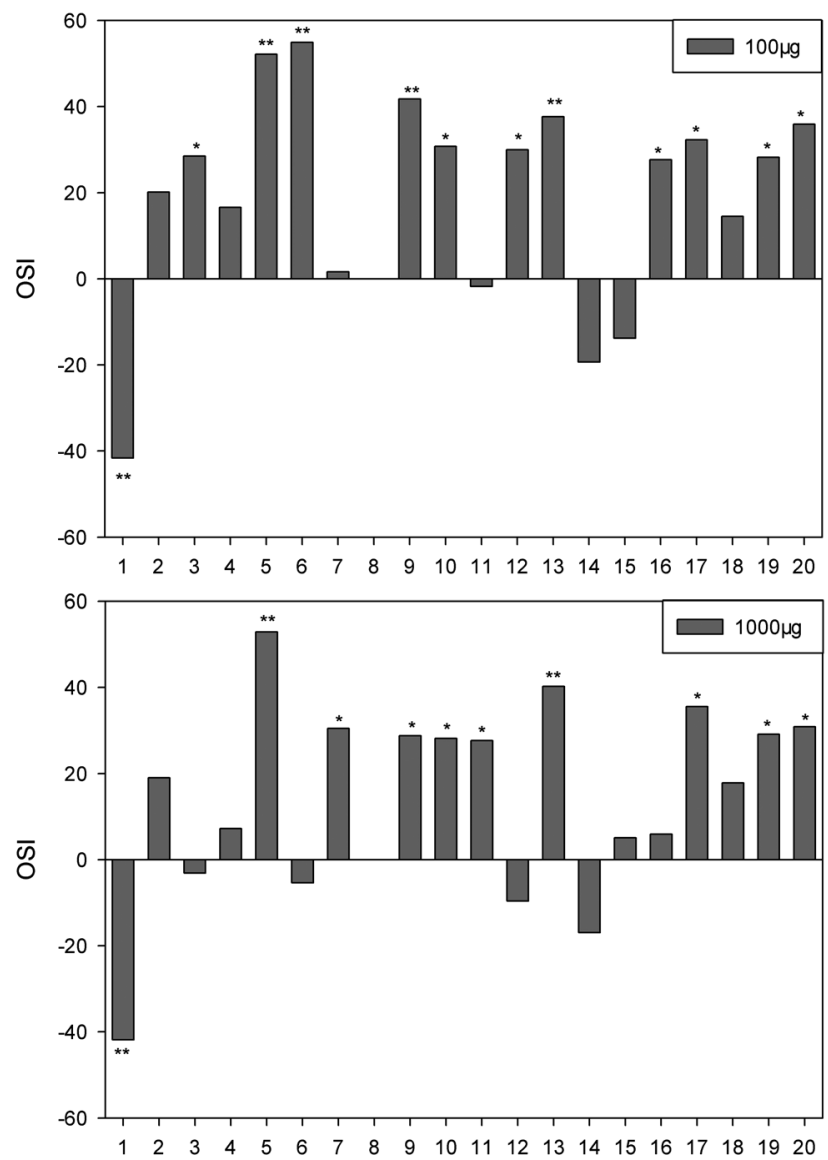

Fig. 2. Oviposition responses of Ostrinia furnacalis females to different compounds rinsed from leaf surfaces. X-axis indicates the chemicals using the same numbers as in figure $1 . *$ Significant differences between the number of eggs on the treated and on the control side using $\chi^{2}$ tests $(P<0.05)$. ${ }^{* *} P<0.01$. The oviposition stimulation index (OSI) is on the Y-axis.

$\left.\mu \mathrm{g}: \chi^{2}=4.049, P=0.044\right),(\mathrm{Z})-9$-octadecenamide $(100 \mu \mathrm{g}$ : $\left.\chi^{2}=7.344, P=0.007 ; 1,000 \mu \mathrm{g}: \chi^{2}=8.468, P=0.004\right)$, heptacosane $\left(100 \mu \mathrm{g}: \chi^{2}=5.343, P=0.021 ; 1,000 \mu \mathrm{g}: \chi^{2}\right.$ $=6.536, P=0.011)$, octacosane $\left(100 \mu \mathrm{g}: \chi^{2}=4.065, P=\right.$ 0.044; $\left.1,000 \mu \mathrm{g}: \chi^{2}=4.325, P=0.038\right)$ and nonacosane $\left(100 \mu \mathrm{g}: \chi^{2}=6.666, P=0.010 ; 1,000 \mu \mathrm{g}: \chi^{2}=4.892, P=\right.$ $0.027)$ were attractive to female moths at concentrations of both $1,000 \mu \mathrm{g}$ and $100 \mu \mathrm{g}$. Hexadecane $\left(\chi^{2}=4.144, P=\right.$ $0.042)$, octadecane $\left(\chi^{2}=16.326, P=0.000\right)$, tricosane $\left(\chi^{2}=\right.$ 4.596, $P=0.032)$, hexacosane $\left(\chi^{2}=3.897, P=0.048\right)$ were attractive at a concentration of $100 \mu \mathrm{g}$, while hexadecanoic acid methyl ester $\left(\chi^{2}=4.759, P=0.029\right)$ and docosane $\left(\chi^{2}\right.$ $=3.889, P=0.049)$ was attractive at $1,000 \mu \mathrm{g}$. The largest OSI was recorded for tetradecanoic acid at a concentration of $5 \times 10^{-3} \mathrm{~g} / \mathrm{mL}$ (Fig. 2). But decanal, heptadecane and (Z)-13-docosenamide did not differ significantly from the control group at concentrations of both $100 \mu \mathrm{g}$ and 1,000 $\mu g$ (Fig. 2).

They were repelled by nonanal at concentrations of $5 \times 10^{-3} \mathrm{~g} / \mathrm{mL}\left(\chi^{2}=9.165, P=0.002\right)$ and $5 \times 10^{-4} \mathrm{~g} / \mathrm{mL}$ $\left(\chi^{2}=9.051, P=0.003\right)$. The OSI of $1,000 \mu \mathrm{g}$ nonanal was -41.86 , which was significantly less than that of the control group (Fig. 2). Similar repellant results were also ob- 
tained for docosane, pentacosane and tetracosane at a dose of $100 \mu \mathrm{g}$ and for hexadecane, octadecane, tricosane and tetracosane at a dose of $1,000 \mu \mathrm{g}$, but they did not differ significantly from their control groups (Fig. 2).

\section{DISCUSSION}

The antennae of $O$. furnacalis responded to 21 compounds present in the leaf surfaces of maize and sugarcane. Oviposition bioassays used in the present study indicate that females of $O$. furnacalis females respond to similar chemicals in maize and sugarcane. This result is consistent with a recent report from our laboratory that crude extracts of both maize and sugarcane leaves attract ovipositing females (Jiang et al., 2014). Similar oviposition responses to epicuticular chemicals are recorded for $O$. nubilalis females (Udayagiri \& Mason, 1997).

Plant volatiles differ in intercropping systems and affect the orientation, mating, oviposition and migration of pests (Dong et al., 2013). To the best of our knowledge, this study is the first to report that compounds in surfaces of leaves in sugarcane-maize intercropping systems influence oviposition by $O$. furnacalis. The similarity of the compounds rinsed from surfaces of the leaves of the two plants accounts for their similarity in attracting ovipositing females of $O$. furnacalis and is in accordance with chemicals playing a crucial role in insect oviposition behaviour (Zhang \& Ji, 1994).

In total $74.14 \%$ of the compounds were recorded in the surface of leaves of both sugarcane and maize, which were classified from the same family that generate similar guild of compounds. The similarity of the compounds in both the main crop and trap crop in the intercropping system studied, indicate they could act as chemical cues and distract $O$. furnacalis from laying eggs on maize leaves (Jiang et al., 2014). 21 compounds were identified that were detected by the antennae of $O$. furnacalis (Fig. 1). These compounds are: aldehydes (nonanal, decanal), acids (tetradecanoic acid, (Z)-7-hexadecenoic acid, hexadecanoic acid and octadecanoic acid), esters (hexadecanoic acid methyl ester), amides ((Z)-9-octadecenamide and (Z)-13-docosenamide) and hydrocarbons (from $\mathrm{C}_{16}$ to $\mathrm{C}_{18}$, and $\mathrm{C}_{22}$ to $\mathrm{C}_{29}$ ).

The aldehydes, such as nonanal and decanal, were EADactive components for $O$. furnacalis, which is in accordance with a previous study that indicates that nonanal inhibit oviposition by gravid females (Huang et al., 2009).

The acids [tetradecanoic acid, (Z)-7-hexadecenoic acid, hexadecanoic acid and octadecanoic acid] and their methyl esters are both present in larval frass in sugarcane-maize intercropping systems. The dual functions of these acids and their methyl esters in larval frass and maize-sugarcane intercropping volatiles are dose-dependent. Total amount of the fatty acids produced by $O$. furnacalis and the other three species of Ostrinia range between 110-180 $\mu \mathrm{g} / 100$ mg of fresh frass (Li \& Ishikawa, 2004). The high concentrations of acids and esters in larval frass deter O. furnacalis from ovipositing ( $\mathrm{Li} \&$ Ishikawa, 2004; Guo \& Li, 2009), which is in accordance with the results of a study on O. nubilalis (Ditrick et al., 1983). In the present study, the concentration in the sugarcane-maize intercropping system was only $5 \times 10^{-3} \mathrm{~g} / \mathrm{mL}$ or $5 \times 10^{-4} \mathrm{~g} / \mathrm{mL}$, respectively. The concentration of acids and esters present in the surfaces of both sugarcane and maize leaves is attractive to ovipositing females of $O$. furnacalis and accounts for the success of intercropping sugarcane with maize in China as means of controlling this pest (Bao \& Lin, 2011; Jiang et al., 2014).

Amides, such as (Z)-9-octadecenamide and (Z)-13-docosenamide, were first reported to be present among the compounds rinsed from the surfaces of leaves of both sugarcane and maize. The OSI of $O$. furnacalis was less than 0 for (Z)-9-octadecenamide and greater than 0 for (Z)-13-docosenamide; however, the OSIs do not differ significantly.

Hydrocarbons that act as oviposition stimulants for $O$. furnacalis are present in both sugarcane and maize, and as a consequence $O$. furnacalis females often lay eggs on the wrong host plant in sugarcane and maize intercropping systems, which results in few of their larvae surviving because for them sugarcane is inedible (Jiang et al., 2014). This is consistent with previous studies on the oviposition of $O$. nubilalis, which is elicited by chemicals in the epicuticle of the leaves of maize, such as hexacosane, heptacosane, octacosane and nonacosane (Udayagiri \& Mason, 1997).

Consequently, this research indicates that the compounds in the leaf surfaces of sugarcane induce $O$. furnacalis females to oviposit because most of them are similar to those in leaf surfaces of maize. This would reduce the incidence of egg masses laid on maize leaves. Therefore, maize plants may be largely protected from damage by $O$. furnacalis by using a sugarcane-maize intercropping system.

ACKNOWLEDGEMENTS. We first would like to thank the EJE editor and anonymous reviewer for their kind help to improve this manuscript. We are grateful to Z. Yu (Kunming Institute, Botanic Garden, Chinese Academy of Sciences) for technical analysis of leaf surface compounds of sugarcane and maize using GC-MS. This research was funded by grants from the National Basic Research Program of China (973 foundation, No. 2011CB100404) and National Natural Science Fund of China (No. 31160363, No. 30860069). We are also grateful to the following graduate students X. Xie, X. Li, X. Chen and Z. Yang for their help with rearing the insects and plants. We also want to thank J. Teichroew, a native English speaker, who improved our English. The authors declare that no competing interests exist.

\section{REFERENCES}

BAO J.A. \& Lin L. 2011: Feasibility and key technologies of intercropping sugarcane with fresh corn. - J. Guangxi Agric. 26: 29-35 [in Chinese, English abstr.].

Calumpang S.M.F. \& Navasero M.V. 2013: Behavioral response of the Asian corn borer Ostrinia furnacalis Guenée (Lepidoptera: Pyralidae) and the earwig Euborelia annulipes Lucas (Dermaptera: Anisolabiidae) to selected crops and weeds associated with sweet corn. - Philipp. Agric. Sci. 96: 48-54.

Cook S.M., Khan Z.R. \& Pickett J.A. 2007: The use of pushpull strategies in integrated pest management. - Annu. Rev. Entomol. 52: 375-400.

Ditrick L., Jones R. \& Chiang H. 1983: An oviposition deterrent for the European corn borer, Ostrinia nubilalis (Lepidoptera: Pyralidae), extracted from larval frass. — J. Insect Physiol. 29: 119-121. 
Dong W.X., Xu N. \& XIAo C. 2013: The effect of diversified cropping on phytophagous insect behavior. - Chin. J. Appl. Entomol. 50: 1133-1140 [in Chinese, English abstr.].

Eaglesham A., Ayanaba A., Rao V.R. \& EsKew D. 1981: Improving the nitrogen nutrition of maize by intercropping with cowpea. - Soil Biol. Biochem. 13: 169-171.

Guo L. \& Li G.Q. 2009: Olfactory perception of ovipositiondeterring fatty acids and their methyl esters by the Asian corn borer, Ostrinia furnacalis. - J. Insect Sci. 9: 1-9.

He K.L., Wang Z.Y., Bai S.X., Zheng L., Wang Y.B. \& Cui H.Y. 2006: Efficacy of transgenic Bt cotton for resistance to the Asian corn borer (Lepidoptera: Crambidae). - Crop. Prot. 25: $167-173$.

Huang C.H., Yan F.M., Byers J.A., Wang R.J. \& Xu C.R. 2009: Volatiles induced by the larvae of the Asian corn borer ( $\mathrm{Os}_{-}$ trinia furnacalis) in maize plants affect behavior of conspecific larvae and female adults. - Insect Sci. 16: 311-320.

JaVAnMard A., Nasab A.D.M., Javanshir A., Moghaddam M. \& JANMOHAMMADI H. 2009: Forage yield and quality in intercropping of maize with different legumes as double-cropped. $-J$. Food Agric. Environ. 7: 163-166.

Jellum E. \& Kuo S. 1996: Nitrogen requirements of corn (Zea mays L.) as affected by monocropping and intercropping with alfalfa (Medicago sativa). — Nutr. Cycl. Agroecosyst. 47: 149156.

Jiang X.C., Xie X.W., Li X., Dong W.X., Xiao C., Chen B., Yan N.S. \& Li Z.Y. 2014: Effect of sugarcane and corn odors on adult oviposition and larvae feeding behaviors of Asian corn borer (Ostrinia furnacalis). - Chin. J. Appl. Entomol. 51: 615-622 [in Chinese, English abstr.].

Khan Z.R., Ampong-Nyarko K., Chiliswa P., Hassanali A., Kimani S., Lwande W., Overholt W.A., Pickett J.A., Smart L.E. \& Woodcock C.M. 1997: Intercropping increases parasitism of pests. - Nature 388: 631-632.

Khan Z.R., Pickett J.A., Berg J., Wadhams L.J. \& Woodcock C.M. 2000: Exploiting chemical ecology and species diversity: stem borer and striga control for maize and sorghum in Africa. — Pest Manag. Sci. 56: 957-962.

Konstantopoulou M., Krokos F. \& Mazomenos B. 2004: Chemical composition of corn leaf essential oils and their role in the oviposition behavior of Sesamia nonagrioides females. - J. Chem. Ecol. 30: 2243-2256.

LI G. \& IsHIKAWA Y. 2004: Oviposition deterrents in larval frass of four Ostrinia species fed on an artificial diet. - J. Chem. Ecol. 30: $1445-1456$.

Li C.Y., He X.H., Zhu S.S., Zhou H.P., WANG Y.Y., Li Y., YANG J., FAN J.X., YANG J.C., WANG G.B., Long Y.F., XU J.Y., TANG Y.S., Zhao G.H., YANG J.R., Liu L., Sun Y., XIE Y., Wang H.N. \& Zнu Y.Y. 2009: Crop diversity for yield increase. - PLoS One. 4: e8049.

Litsinger J.A., Hasse V., Barrion A.T. \& Schmutterer H. 1991: Response of Ostrinia furnacalis (Guenée) (Lepidoptera: Pyralidae) to intercropping. - Environ. Entomol. 20: 988-1004.
Lu P., HuANG L. \& WANG C. 2012: Identification and field evaluation of pear fruit volatiles attractive to the oriental fruit moth, Cydia molesta. - J. Chem. Ecol. 38: 1003-1016.

NAFus D. \& SCHREINER I. 1991: Review of the biology and control of the Asian corn borer, Ostrinia furnacalis (Lep.: Pyralidae). - Int. J. Pest. Manag. 37: 41-56.

Poveda K. \& Kessler A. 2012: New synthesis: plant volatiles as functional cues in intercropping systems. $-J$. Chem. Ecol. 38: 1341 .

Rea J.H., Wratten S.D., Sedcole R., Cameron P.J., Davis S.I. \& Chapman R.B. 2002: Trap cropping to manage green vegetable bug Nezara viridula (L.) (Heteroptera: Pentatomidae) in sweet corn in New Zealand. - Agr. Forest. Entomol. 4: 101-107.

Risch S.J., Andow D. \& Altieri M.A. 1983: Agroecosystem diversity and pest control: data, tentative conclusions, and new research directions. - Environ. Entomol. 12: 625-629.

RUther J. \& KleIER S. 2005: Plant-plant signaling: ethylene synergizes volatile emission in Zea mays induced by exposure to (Z)-3-hexen-1-ol. - J. Chem. Ecol. 31: 2217-2222.

Shelton A. \& Badenes-Perez F. 2006: Concepts and applications of trap cropping in pest management. - Annu. Rev. Entomol. 51: $285-308$.

Steinbauer M.J., Schiestl F.P. \& Davies N.W. 2004: Monoterpenes and epicuticular waxes help female autumn gum moth differentiate between waxy and glossy Eucalyptus and leaves of different ages. - J. Chem. Ecol. 30: 1117-1142.

UDAYAGIRI S. \& MASON C.E. 1995: Host plant constituents as oviposition stimulants for a generalist herbivore: European corn borer. - Entomol. Exp. Appl. 76: 59-65.

UdAYAGIRI S. \& MASON C.E. 1997: Epicuticular wax chemicals in Zea mays influence oviposition in Ostrinia nubilalis. - J. Chem. Ecol. 23: 1675-1687.

Zehnder G., Gurr G.M., Kühne S., Wade M.R., Wratten S.D. \& WYsS E. 2007: Arthropod pest management in organic crops. - Annu. Rev. Entomol. 52: 57-80.

Zhang Q.H. \& JI L.Z. 1994: Chemical ecology of oviposition of herbivorous insect. - Chin. J. Ecol. 13: 39-43 [in Chinese, English abstr.].

Zhou D.R., He K.L., Wang Z.Y., Ye Z.H., Wen L.P., Gao Y.X. \& SoNG Y.Y. 1995: Asian Corn Borer and its Integrated Management. Golden Shield Press, Beijing, 102 pp. [in Chinese].

ZHOU D.R., WANG Z.Y. \& LIU B.L. 1980: Research on raising Asian corn borer in large scale: I. A kind of semi-artificial diet and its improvement. - Acta Phytophylac. Sin. 7: 113-122 [in Chinese, English abstr.].

Zhu Y.Y., Chen H.R., FAN J.H., WANG Y.Y., Li Y., Chen J.B., FAN J.X., YAng S.S., Hu L.P. \& Leung H. 2000: Genetic diversity and disease control in rice. - Nature 406: 718-722.

Received June 16, 2014; revised and accepted October 13, 2014 Prepublished online February 16, 2015 
